\title{
Lidamycin inhibits tumor growth and pulmonary metastasis in murine breast carcinoma and shows synergy with paclitaxel
}

\author{
HU Lei, ZHANG ShengHua, SHAO RongGuang \& ZHEN YongSu ${ }^{*}$ \\ Institute of Medicinal Biotechnology, Chinese Academy of Medical Sciences \& Peking Union Medical College, Beijing 100050, China
}

Received May 23, 2012; accepted July 12, 2012; published online May 9, 2013

\begin{abstract}
The main goal of this study was to investigate whether lidamycin (LDM) could enhance the efficacy of paclitaxel (TAX) against breast cancer. In the MTT assay, LDM showed much more potent cytotoxicity than paclitaxel, and there was a synergy on the 4T1/luc breast cancer cells treated with a combination of paclitaxel and LDM. Western blot analysis showed that paclitaxel and LDM synergistically downregulated MMP9, MMP2, VEGF, and upregulated the cleaved PARP proteins. By wound closure cell migration assay, paclitaxel combined LDM obviously inhibited the migration of 4T1/luc cells. At therapeutic dosage level, LDM, paclitaxel, and the combination suppressed the pulmonary metastases by $70.2 \%, 53.8 \%$, and $88.7 \%$, respectively, and the CDI value was 0.82 , indicating synergism. The results show that LDM enhances the antitumor effect of paclitaxel on 4T1/luc breast cancer, in particular, the antimetastatic effects on pulmonary metastasis.
\end{abstract}

lidamycin, paclitaxel, breast cancer, antitumor, antimetastatic

Citation: Hu L, Zhang S H, Shao R G, et al. Lidamycin inhibits tumor growth and pulmonary metastasis in murine breast carcinoma and shows synergy with paclitaxel. Chin Sci Bull, 2013, 58: 2805-2811, doi: 10.1007/s11434-013-5732-3

Breast cancer has been the second leading cause of cancer-related mortality among women throughout the world [1]. Surgery is still the mainstay of treatment for primary breast cancer, but is ineffective for metastatic tumors at distant sites, and it may paradoxically increase the dissemination of tumor cells into the bloodstream resulting in the seeding of tumor cells in distant organs [2,3]. Adjuvant chemotherapy for breast cancer after surgery has been the standard of care, and can effectively prevent the occurrence of tumor cell dissemination and metastasis. Paclitaxel (Taxol, TAX) has been proved to be a potent drug to treat metastatic breast cancer, ovarian cancer, and other forms of cancer [4]. It blocks the cell cycle in the G2 and M phase by preventing polymerization of microtubules, thereby causing toxicity and cell death [5]. Paclitaxel is active in the treatment of metastatic breast cancer as first-line therapy [6], as well as in heavily pretreated patients [7,8]. Especially encouraging is its activity in anthracycline-resistant disease

\footnotetext{
*Corresponding author (email: zhenys@ public.bta.net.cn)
}

[9]. However, several toxic side effects and clinical implications, including severe anaphylactic peripheral neuropathy and hypersensitivity reactions, have been reported in paclitaxel-treated patients. Therefore, potential therapeutic strategies to improve the efficacy of paclitaxel and reducing its side effects are needed.

Anticancer antibiotic lidamycin (LDM, C-1027) was screened from the broth filtrate of Streptomyces globisporus C-1027 by spermatogonial assay. Strain C-1027 was isolated from a soil sample collected in Qianjiang area, Hubei Province of China [10]. LDM belongs to a family of enediyne antibiotics and shows extremely potent cytotoxicity by causing DNA double-strand breaks. LDM also markedly inhibits the growth of transplantable tumors in vivo [11-13]. LDM has demonstrated unique characteristics in the treatment of cancer, such as preferentially targeting hypoxic cells, dosage-dependent regimens based upon p53 status, and high activity against multidrug-resistant cancer cells [14-16]. LDM is now in phase II clinical trials as a potential chemotherapeutic agent in China. 
Recent study revealed that LDM combined with other anticancer drugs can effectively enhance the antitumor activity $[17,18]$. In this study, the highly metastatic $4 \mathrm{~T} 1$ breast cancer model was used. As well known, the 4T1 cell line was derived from a spontaneously arising $\mathrm{BLAB} / \mathrm{c}$ mammary tumor. It grows progressively and causes a uniformly lethal disease even after excision of the primary tumor [19]. 4T1 tumor metastasizes via the hematogenous route to the liver, lungs, bone, and the brain. It closely resembles stage IV human breast cancer both in its immunogenicity, growth and metastatic properties, making it an excellent animal model to evaluate the effects of drugs on breast tumor metastases as well as tumor growth [20-22]. Herein, we investigated whether the combination of LDM with paclitaxel would enhance the antitumor and antimetastatic activity to combat the highly metastatic $4 \mathrm{~T} 1$ breast cancer in vitro and in vivo.

\section{Materials and methods}

\subsection{Cell culture}

The mouse breast cancer cell line 4T1 was purchased from ATCC (ATCC number CRL-2539), and the cell line 4T1/ luc was constructed by stable expression of firefly luciferase. The 4T1/luc cells showed an equivalent metastatic potential with the parental 4T1 cells. The cells were cultured in RPMI-1640 (Gibco, USA) supplemented with 10\% FBS (Gibco), $100 \mathrm{U} \mathrm{mL}^{-1}$ penicillin, and $100 \mu \mathrm{g} \mathrm{mL}^{-1}$ streptomycin, 2 mmol L ${ }^{-1}$ L-glutamine and $400 \mu \mathrm{g} \mathrm{mL}^{-1} \mathrm{G} 418$ in a humidified atmosphere of $5 \% \mathrm{CO}_{2}$ in air.

\subsection{Transfection and selection of stably transfected 4T1/luc cells}

4T1/luc cells at 70\%-80\% confluence were transfected with $1 \mu \mathrm{g}$ of luciferase expression plasmid PCAGGS-NEO-luc using Lipofectamine 2000 (Invitrogen, Carlsbad, CA, USA) according to the manufacturer's instructions. For stable transfection, the cells were exposed to $500 \mu \mathrm{g} \mathrm{mL}^{-1} \mathrm{G} 418$ (Gibco) after transfection 1 day later. After growth for 3 weeks, cells were plated at a lower density in RPMI- 1640 with $500 \mu \mathrm{g} \mathrm{mL}^{-1} \mathrm{G} 418$ and $10 \%$ FBS in 96-wells plates until single colony was formed. Single cloned cells were isolated and expanded. To identify the positive clones, monoclonal cells were plated in 96-well plates and luciferase substrate D-luciferin was added to a final concentration of $60 \mathrm{mg} \mathrm{mL} \mathrm{m}^{-1}$. After $30 \mathrm{~min}$ of exposure, the fluorescence intensity of expression was examined with the optical imaging system (IVIS 200, Xenogen) and the most fluorescent clone was selected and cryopreserved.

\subsection{Luciferase activity test of 4T1/luc cells in vitro}

The 4T1/luc cells were diluted in 96 well plates at a density of $6000,3000,1500,750,300,150$, and 75 cells per well, respectively, and the cells were exposed to the fluorescent luciferase substrate D-luciferin. The luminous intensity was detected in the camera box by IVIS-Imaging System.

\subsection{Cell viability assay}

Cell viability was evaluated by using the MTT assay. 4T1/luc cells were seeded in 96-well plates (Costar, USA) at a density of $3 \times 10^{3}$ cells/well in $200 \mu \mathrm{L}$ medium. The next day cells in triplicate wells were treated with LDM $\left(10^{-14}\right.$, $\left.10^{-13}, 10^{-12}, 10^{-11}, 10^{-10}, 10^{-9}, 10^{-8}, 10^{-7} \mathrm{~mol} \mathrm{~L}^{-1}\right)$, paclitaxel $\left(10^{-11}, 10^{-10}, 10^{-9}, 10^{-8}, 10^{-7}, 10^{-6}, 10^{-5}, 10^{-4} \mathrm{~mol} \mathrm{~L}^{-1}\right)$, or the combination of the two agents. After drug exposure for $48 \mathrm{~h}, 20 \mu \mathrm{L}$ MTT solutions $\left(5 \mathrm{mg} \mathrm{mL}^{-1}\right)$ were added to each plate. After being incubated at $37^{\circ} \mathrm{C}$ for another $4 \mathrm{~h}$, the formazan, which is derived from MTT by living cells, was dissolved in $150 \mu \mathrm{L}$ per well of DMSO, and the absorbance was detected at $570 \mathrm{~nm}$. The percentage of cell viability was calculated as follows: cell viability $(\%)=\left(\mathrm{OD}_{570}\right.$ of experimental well-OD ${ }_{570}$ of blank $) /\left(\mathrm{OD}_{570}\right.$ of control well-OD ${ }_{570}$ of blank). The $\mathrm{IC}_{50}$ (defined as the drug concentration with which $50 \%$ cell growth was inhibited) was assessed from the dose-response curves. All MTT experiments were performed in triplicate and repeated at least 3 times.

\subsection{Western blot analysis}

Cells treated with LDM (0.01 nmol $\left.\mathrm{L}^{-1}\right)$, paclitaxel (10

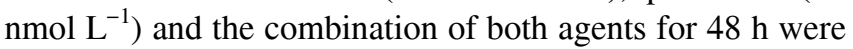
lysed in RIPA buffer on ice and then centrifuged at 12000 $\mathrm{r} \mathrm{min}{ }^{-1}$ for $10 \mathrm{~min}$ at $4^{\circ} \mathrm{C}$ to collect the supernatant. Protein concentrations were determined using BCA reagent (Pierce, USA). Protein samples were separated by $12 \%$ SDS-PAGE and than transferred to a polyvinylidene membrane. After incublated overnight at $4{ }^{\circ} \mathrm{C}$ in $1 \%$ BSA-PBST(phosphate buffered saline contained $0.1 \%$ Tween 20 ), the membranes were incublated with primary and secondary antibodies, band intensity was detected by chemiluminescence using ECL detection reagents (Millipore, USA). Blots were stained with an anti- $\beta$-actin to confirm that each lane contained similar amounts of tumor homogenate.

\subsection{Wound closure cell migration assay}

4T1/luc cells were seeded in a 6-well plate and incublated $24 \mathrm{~h}$ to allow for confluency. A $10 \mu \mathrm{L}$ pipette tip was then used to create a wound through the center of the confluent cell layer, and the size of the wound was measured. Cells were then treated with $3 \mathrm{~mL}$ LDM $\left(0.01 \mathrm{nmol} \mathrm{L}^{-1}\right)$, paclitaxel $\left(10 \mathrm{nmol} \mathrm{L}^{-1}\right)$ and the combination of both agents for $24 \mathrm{~h}$. Photos were taken of the wound periodically throughout the assay. To quantify the changes, the size of the wound was measured again after $24 \mathrm{~h}$. This analysis gave the distance $(\mu \mathrm{m})$ that tumor cells migrated. 


\subsection{Animal experiments}

The 6-8-week-old female BALB/c mice were purchased from the Institute of laboratory Animal Sciences, Chinese Academy of Medical Sciences \& Peking Union Medical College. 4T1/luc cells in log phase were suspended to a final concentration of $5.0 \times 10^{6}$ cells $\mathrm{mL}^{-1}$ with phosphatebuffered saline (PBS), and $5.0 \times 10^{5} 4 \mathrm{~T} 1 / \mathrm{luc}$ cells in $0.1 \mathrm{~mL}$ were injected into the second mammary fatpad. Primary tumors were measured every 3 days. When tumors reached about $400 \mathrm{~mm}^{3}$ in size, the mice were randomized divided into four groups ( $n=10$ per group). Then the treatment were started, the mice were injected intravenously with LDM $\left(0.05 \mathrm{mg} \mathrm{kg}^{-1}\right)$, intraperitoneally with paclitaxel $\left(5 \mathrm{mg} \mathrm{kg}^{-1}\right)$, and the combination of LDM $\left(0.05 \mathrm{mg} \mathrm{kg}^{-1}\right)$ plus paclitaxel $\left(5 \mathrm{mg} \mathrm{kg}^{-1}\right)$. LDM and paclitaxel were injected once a week and last for two weeks. Control group was injected with saline. Tumor growth was measured with a vernier caliper, and tumor volumes were calculated with the following formula: $V=0.5 a \times b^{2}$, where $a$ and $b$ are the long and the perpendicular short diameters of the tumors, respectively. At day 30, all mice were weighed and sacrificed, and tumors were excised. Tumors were weighed, and the mean tumor weight was calculated. Tumor growth inhibition was calculated by the following formula: $[(C-T) / C]$ $\times 100(C$, tumor weight of control; $T$, tumor weight of treated group). The lungs were removed, and fixed in formalin overnight before evaluation of lung metastasis. The number of lung metastases on the lung surface was counted with a magnifying lens.

The tumor growth inhibitory effect was estimated using the treated/control ratio $(T / C)$. In order to calculate the coefficient of drug interaction (CDI), the following equation was used: $\mathrm{CDI}=A B / A \times B$, where $A$ or $B$ was the mean $T / C$ of drug $A$ and drug $B$ respectively, and $A B$ was the mean $T / C$ of drug A combined with drug B. A CDI greater than 1 indicates antagonism, a CDI equal to 1 indicates additive and a CDI less than 1 indicates synergy.

\subsection{Optical imaging and histological analysis}

At day 30, before sacrificed the mice, an optical molecular imaging system was used to evaluate the growth of primary tumor. Luciferase substrate D-luciferin $\left(150 \mathrm{mg} \mathrm{kg}^{-1}\right)$ was injected intraperitoneally, and the animals were placed onto the warmed stage inside the camera box of IVIS-Imaging System (Xenogen) to observe the tumor and the lungs from different groups. Tissues were fixed in $4 \%$ paraformaldehyde in PBS, embedded in paraffin and cut into 3-5 $\mu \mathrm{m}$ sections through the center of the tissue specimen. Then the sections were stained with hematoxylin and eosin (H \& E). Stained sections were observed using a microscope.

\subsection{Statistics}

The results were presented as the mean \pm SD. A one-way analysis of variance was carried out for multiple comparisons. If there was significant variation between treatment and control groups, the mean values were compared by using Student's $t$-test. $P$-values less than 0.05 were considered statistically significant.

\section{Results}

\subsection{Luciferase activity in 4T1/luc cells}

The bioluminescence photon is positively correlated to the number of cells (Figure 1). The minimum detectable cell number was 750 cells per well according to our results.

\subsection{Effect of LDM in combination with paclitaxel on the growth of 4T1/luc breast cells in vitro}

Dose-response growth inhibitory effects were observed in the MTT assay, and a significant reduction in growth was observes in cells treated with LDM plus paclitaxel compared to cells treated with LDM or paclitaxel alone (Figure 2). And the $\mathrm{IC}_{50}$ values of LDM and paclitaxel for 4T1/luc cell were $4.61 \times 10^{-1}$ and $3.30 \times 10^{2} \mathrm{nmol} \mathrm{L} \mathrm{L}^{-1}$, respectively. LDM showed about 1000 -fold much more potent cytotoxicity than paclitaxel.

\subsection{Western blot analysis}

Western blot analysis was used to detect the metastasisrelated and apoptosis-related proteins. As shown, the me-

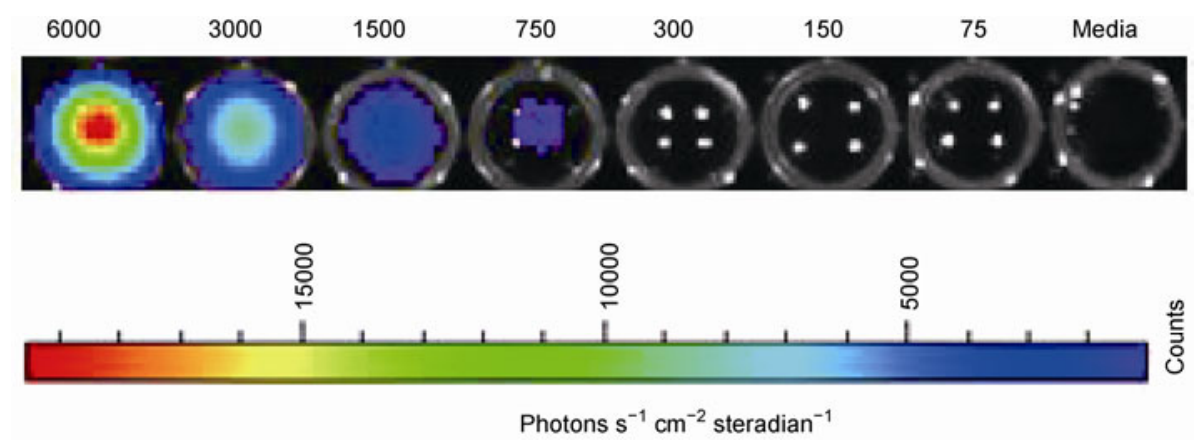

Figure 1 The positive correlation between the photons of bioluminescence and the numbers of 4T1/luc cells. 

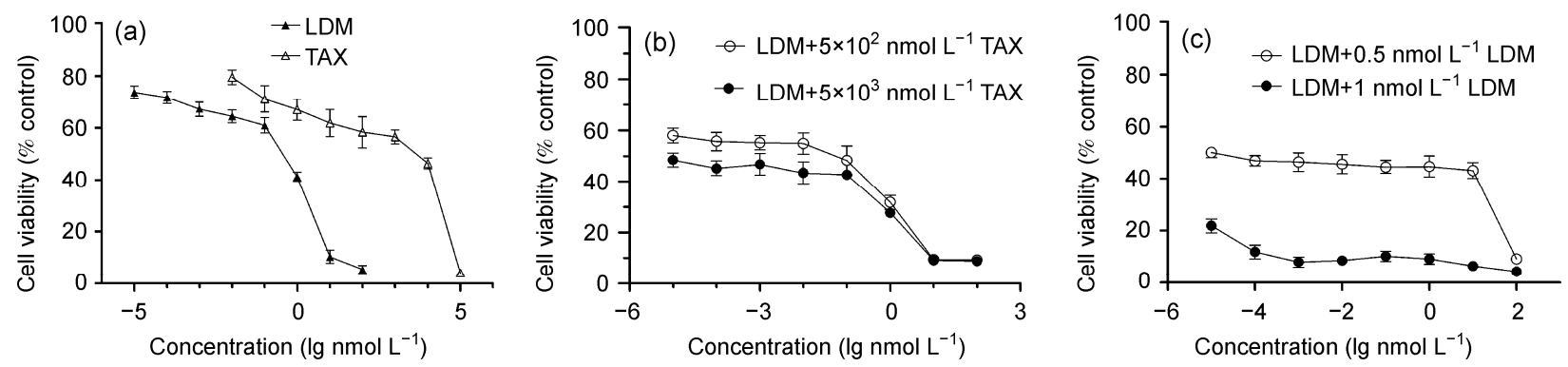

Figure 2 Effect of LDM and paclitaxel on cell viability in 4T1/luc cells. (a) 4T1/luc cancer cells were treated with LDM and paclitaxel alone at concentrations ranging from $10^{-5}$ to $10^{5} \mathrm{nmol} \mathrm{L}{ }^{-1}$ for $48 \mathrm{~h}$. (b) and (c) $4 \mathrm{~T} 1 / \mathrm{luc}$ cells were treated with LDM combined paclitaxel at different concentrations for $48 \mathrm{~h}$.

tastasis-related proteins MMP9, MMP2, VEGF were downregulated, and the combination treatment had a more obvious down-regulation (Figure 3). The $89 \mathrm{kD}$ cleaved fragment of PARP increased after treated. A markedly enhanced effect was observed in the combination group.

\subsection{Wound closure cell migration assay}

To examine the effects of LDM, paclitaxel and the combination on tumor cell migration, the cell wound closure assay was performed. In the wound closure assay (Figure 4), the combined group showed a significant $(P<0.001)$ inhibition of cell migration, compared to the LDM group and paclitaxel group.

\subsection{Animal experiment}

Antitumor and antimetastatic experiments were performed with mouse mammary carcinoma $4 \mathrm{~T} 1 / \mathrm{luc}$ cell in BALB/c mice. In this study, 4T1/luc tumors were growing rapidly in control group in $30 \mathrm{~d}$ during the experiment (Figure 5(a)), and the body weight of each group was recorded (Figure 5(b)). As evaluated on day 30 (Table 1), the LDM and paclitaxel group showed therapeutic efficacy with $38.3 \%$ and $36.5 \%$ TGI, respectively, and the combination group showed higher therapeutic efficacy with $64.2 \%$ TGI for the primary tumor. The CDI value of LDM and paclitaxel was

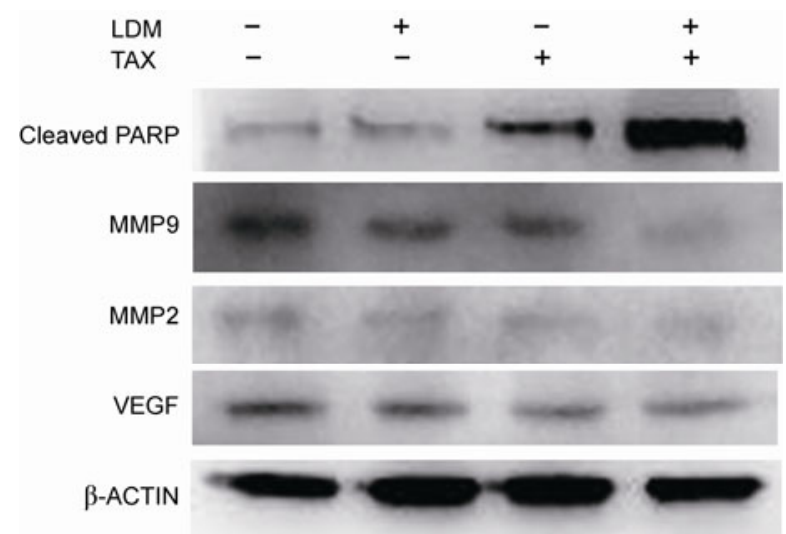

Figure 3 The Western blot analysis of metastasis-related and apoptosisralated proteins.

\subsection{1 , indicating synergism.}

The therapeutic efficacy against metastases was evaluated at day 30 when mice were sacrificed. Lungs were carefully examined. The tumor nodules on the lungs were counted under the dissecting microscope (Figure 6(a) and (c)). Photos of the lungs were taken by IVIS-Imaging System (Figure 6(b) and the pathological changes were observed on sections by HE staining (Figure 6(d)). The combined group significantly reduced the number of lung metastases (Figure 6(b)). The inhibition rates of metastases by LDM, paclitaxel, and the combination of them were $70.2 \%$, $53.8 \%$, and $88.7 \%$, respectively, and the CDI value was 0.82 , indicating synergism.

\section{Discussion}

Metastasis is the most lethal attribute of breast cancer. Once breast cancer metastasizes to distant areas of the body, typically the lung, bones, liver, and central nervous system, it can be treated but is essentially not curable [23]. The 4T1 cell line is widely considered to be one of the best mouse mammary cancer cell lines for the study of human cancer progression [24,25]. This tumor shares many characteristics with human mammary cancers, it closely resembles stage IV human breast cancer, making it an excellent animal model. It was used to investigate inhibitors of DNA-binding proteins necessary for cancer stem cells [26], chemoresistance [27], antimetastatic activities of various drugs and drug combination [28,29]. 4T1/luc cells were engineered for stable expression of firefly luciferase to allow tracking and quantifying of the cells in vivo.

As an enediyne anticancer antibiotic, LDM showed extremely potent cytotoxicity toward cultured cancer cells and markedly inhibited the growth of transplantable tumors in mice [30-32]. LDM (C1027) also showed antiangiogenesis and antimetastatic activity, preferentially targeting hypoxic cells [14]. In the previous study, it has been studied for cancer treatment, enhancement of its targeting, selectivity and efficiency, and drug combination therapy of certain cancers [33-35]. As reported, LDM induced unusual DNA double-strand breaks [36]. It could induce apoptosis or mitotic cell death and alter cell cycle progression in many 
(a)

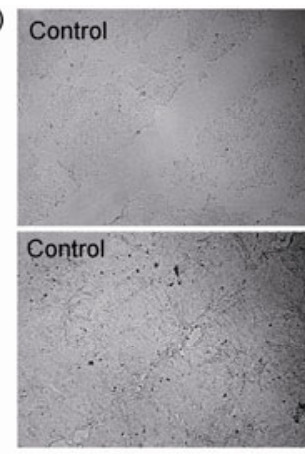

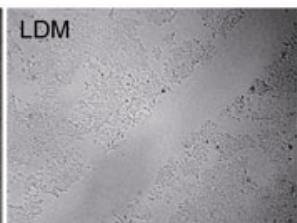

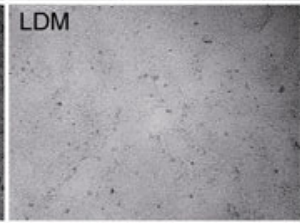

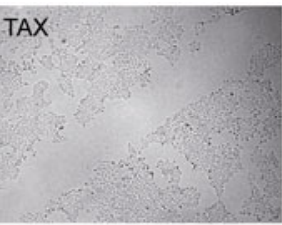
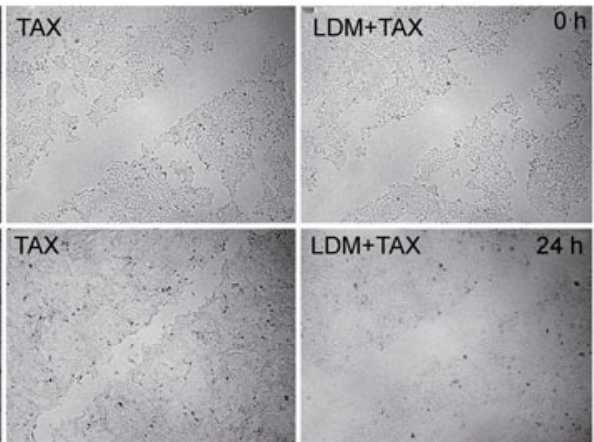

(b)

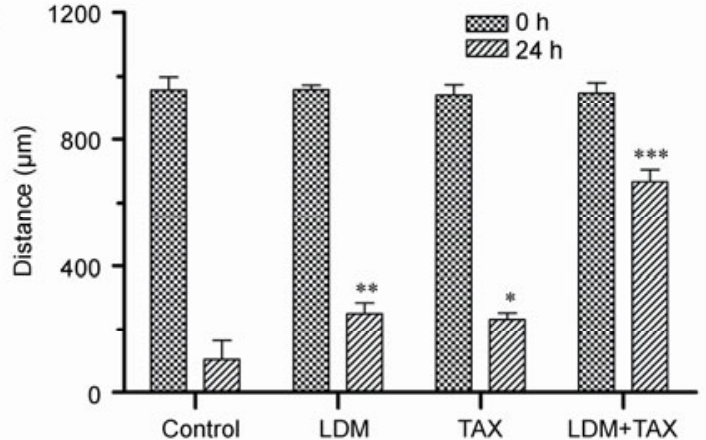

Figure 4 The effects of LDM and paclitaxel on $4 \mathrm{~T} 1 /$ luc tumor cell migration in the wound closure assays. (a) Micrographs of 4T1/luc breast cancer cell wound closure; (b) quantification of 4T1/luc breast cancer cell wound closure. The extent of wound closure was quantified by measuring the distances $(\mu \mathrm{m})$ between the wounds after 24 h. $* P<0.05$ compared to control, $* * P<0.01$ compared to control, *** $P<0.001$ compared to control.
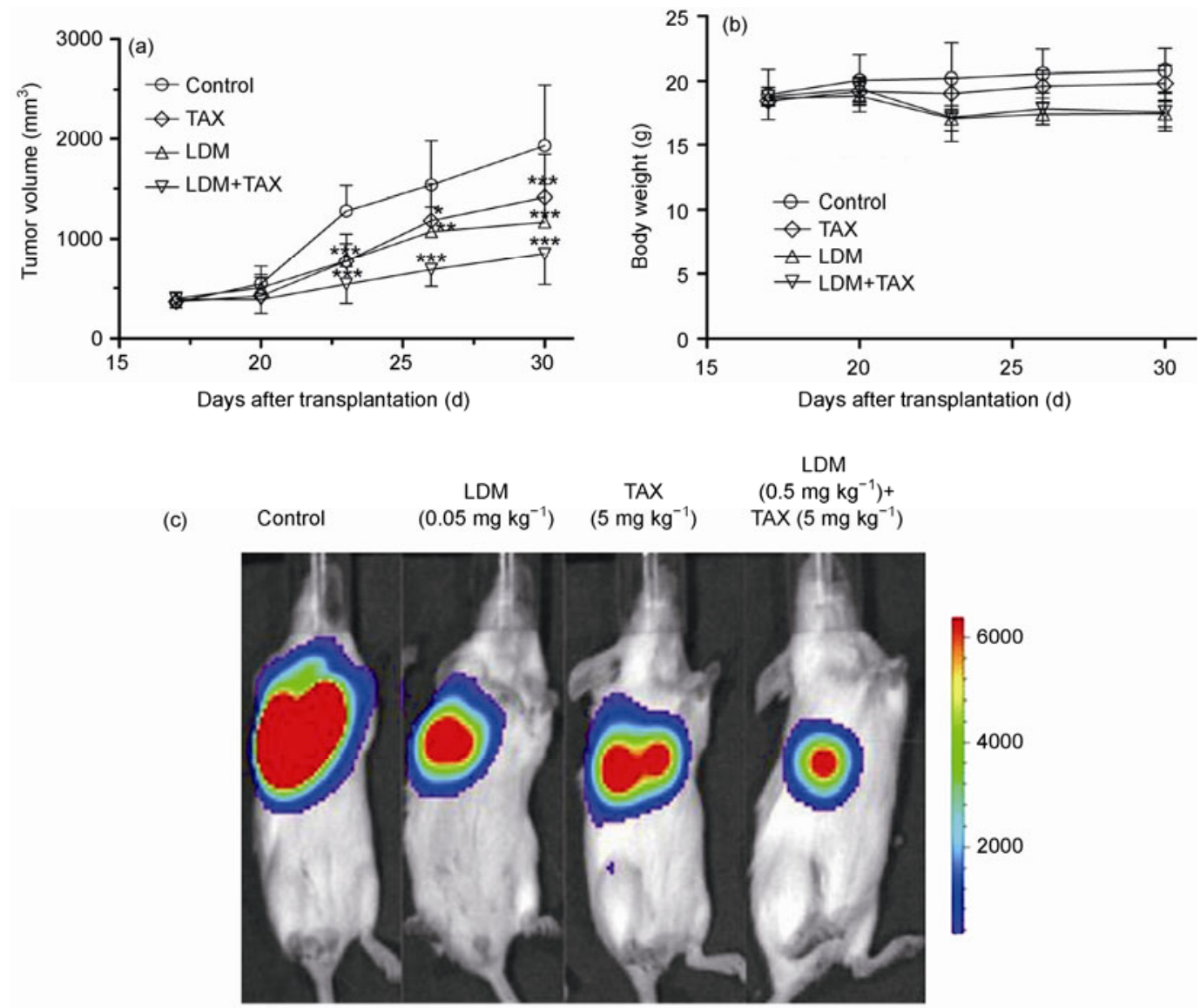

Figure 5 Antitumor activities of LDM, paclitaxel alone or both of them on 4T1/luc breast cancer model. The tumor growth curves (a) and body growth curves (b) in $30 \mathrm{~d}$ duration of experiments. Data are expressed as mean $\pm \mathrm{SD}(n=10)$. (c) Optical imaging in living animal. Color scale represents photons $\mathrm{s}^{-1} \mathrm{~cm}^{-2}$ steradian $^{-1}$, the red dot-cycle indicated the tumor location. $* P<0.05$ compared to control, $* * P<0.01$ compared to control, $* * * P<0.001$ compared to control. 
Table 1 The growth inhibition of mouse mammary carcinoma 4T1/luc primary tumor in BALB/c mice

\begin{tabular}{lccc}
\hline Drug & Dose $\left(\mathrm{mg} \mathrm{kg}^{-1}\right)$ & Number of mice & Tumor weigh $(\mathrm{g})(\mathrm{mean} \pm \mathrm{SD})$ \\
\hline Control & - & $10 / 10$ & $5.36 \pm 1.05$ \\
LDM & 0.05 & $10 / 10$ & $3.31 \pm 0.73$ \\
TAX & 5 & $10 / 10$ & $3.41 \pm 0.80$ \\
LDM+TAX & $0.05+5$ & $10 / 10$ & $38.3^{\mathrm{a})}$ \\
\hline
\end{tabular}

a) $P<0.05$ compared to control; b) $P<0.05$ compared to LDM; c) $P<0.05$ compared to TAX.

(a)

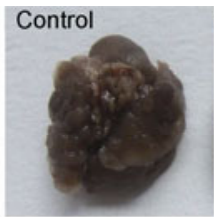

(b)

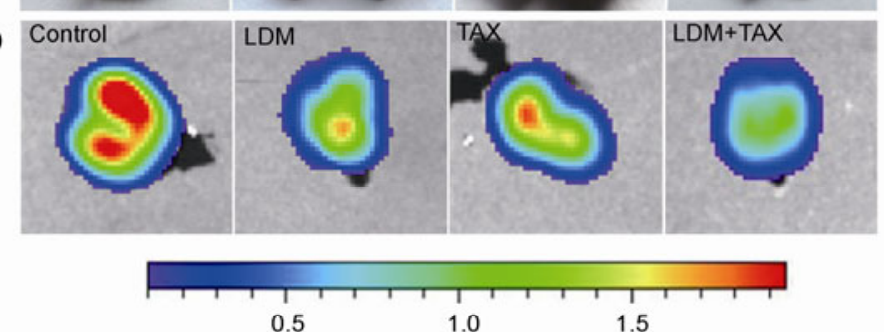

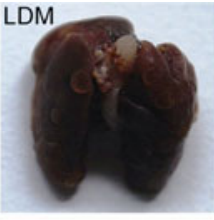

0.5
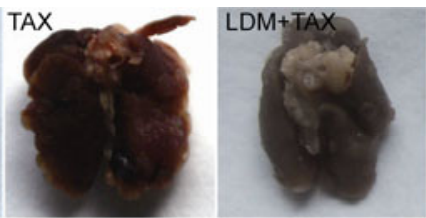

(d)
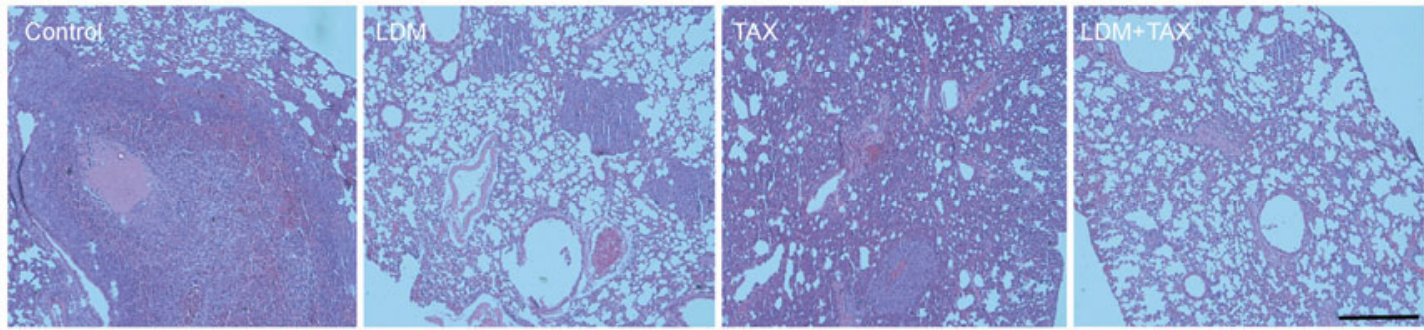

Figure 6 The antimetastatic effect of LDM, paclitaxel, and the combination. (a) Representative photos of the lungs from different groups; (b) optical imaging of lungs from different groups. Color scale represents photons $\mathrm{s}^{-1} \mathrm{~cm}^{-2}$ steradian $^{-1}$. (c) Metastatic nodules on the lung surface. The number of macroscopic metaststic nodules was observed on the lung surface of treated mice killed on day $30(n=10)$. Each data point represents the value for an individual animal. Solid bars represent the mean value for each treatment groups. (d) The pathological changes of lungs by H.E. staining were observed with light microscope $(\times 100)$.

cancer cells [37]. So far, there are no reports describing the combined efficacy of paclitaxel plus LDM on the antitumor and antimetastatic activities with a breast cancer animal model with highly metastatic potential.

In this study, LDM combined paclitaxel exhibited potent antitumor and antimetastatic effects against the highly metastatic mouse $4 \mathrm{~T} 1 /$ luc breast cancer without obvious systemic toxicity. LDM combined paclitaxel resulted an approximately $30 \%$ higher inhibition rate in tumor growth compared to that of LDM, paclitaxel alone. The reduction in lung metastases might be due to: (1) LDM and paclitaxel significantly suppressing the growth of the primary tumors. Smaller and less viable primary tumors would beat less risk of dissemination. (2) LDM and paclitaxel directly preventing the formation of the lung metastases. To our knowledge, this is the first report of detection of LDM and paclitaxel combination effected on a metastatic mouse breast cancer model. Evidently, with the construction of the stable expression of firefly luciferase $4 \mathrm{~T} 1 / \mathrm{luc}$ cell lines, we can monitor and quantitatively analyze the tumor growth and metastasis simply and directly.

In conclusion, LDM or paclitaxel alone exerts antitumor and antimetastatic activity in the highly metastatic mouse breast cancer model. The combination of LDM and paclitaxel synergistically enhances the antitumor and antimetastatic efficacy.

This work was supported by the Significant New Drugs Development Science and Technology Major Projects of China (2010ZX09401-407) and State-level Public Welfare Scientific Research Institutes for Basic R\&D Special Fund (IMBF201101).

1 Jiang $\mathrm{H} \mathrm{C}$, Tao W Y, Zhang $\mathrm{M}$, et al. Low-Dose metronomic paclitaxel chemotherapy suppresses breast tumors and metastases in mice. Cancer Investigation, 2010, 28: 74-84

2 Demichell R, Valagussa P, Bonadonna G. Does surgery modify growth kinetics of breast cancer micrometastases. Br J Cancer, 2001, 85: 490-492

3 Hansen E, Wolff N, Knuechel R, et al. Tumor cells in bloodshed from the surgical field. Arch Surg, 1995, 130: 387-393 
4 Arbuck S G, Dorr A, Friedman M A. Paclitaxel (Taxol) in breast cancer. Hematol Oncol Clin North Am, 1994, 8: 121-140

5 Horwitz S B, Cohen D, Rao S, et al. Paclitaxel: Mechanisms of action and resistance. J Natl Cancer Inst, 1993, 15: 55-61

6 Davidson N G. Single-agent paclitaxel as first-line treatment of metastatic breast cancer: The British experience. Semin Oncol, 1996, 23: 6-10

7 Holmes F A, Valero V, Walters R S, et al. The M.D. Anderson Cancer Center experience with taxol in metastatic breast cancer. J Natl Cancer Inst Monogr, 1993, 15: 161-169

8 Seidman A D, Reichman B S, Crown J P, et al. Paclitaxel as second and subsequent therapy for metastatic breast cancer: Activity independent of prior anthracycline response. J Clin Oncol, 1995, 13: 1152-1159

9 Fountzilas G, Athanassiades A, Giannakakia T, et al. A phase II study of paclitaxel in advanced breast cancer resistant to anthracyclines. Eur J Cancer, 1996, 32: 47-51

10 Hu J L, Xue Y C, Xie M Y, et al. A new macromolecular antitumor antibiotic, C-1027. I. Discovery, taxonomy of producing organism, fermentation and biological activity. Antibiotics, 1988, 41: 1575-1579

11 Shao R G, Zhen Y S. Enediyne anticancer antibiotic lidamycin: Chemistry, biology and pharmacology. Anticancer Agents Med Chem, 2008, 8: 121-131

12 Shao R G, Zhen Y S. Relationship between the molecular composition of $\mathrm{C} 1027$, a new macromolecular antibiotic with enediyne chromophore, and its antitumor activity. Acta Pharmcol Sin, 1995, 30: 336-342

13 Shao R G. Pharmacology and therapeutic applications of enediyne antitumor antibiotics. Curr Mol Pharmacol, 2008, 1: 50-60

14 Beerman T A, Gawron L S, Shin S, et al. C-1027, a radiomimetic enediyne anticancer drug, preferentially targets hypoxic cells. Cancer Res, 2009, 69: 593-598

15 Chen L, Jiang J, Cheng C, et al. P53 dependent and independent apoptosis induced by lidamycin in human colorectal cancer cells. Cancer Biol Therapy, 2007, 6: 965-973

16 Shi Y K, Wu S Y, Huang Y H, et al. Chemosensitivity of mdr1 gene overexpressed multidrug resistant cancer cells to lidamycin (in Chinese). Yao Xue Xue Bao, 2006, 41: 1146-1151

17 Liu H, Li L, Li X Q, et al. Enediyne lidamycin enhances the effect of epidermal growth factor receptor tyrosine kinase inhibitor, gefitinib, in epidermoid carcinoma A431 cells and lung carcinoma H460 cells. Anti Cancer Drugs, 2009, 20: 41-49

18 Chen J, Wu S Y, Ouyang Z G, et al. Synergy of gemcitabine and lidamycin associated with NF-kappaB downregulation in pancreatic carcinoma cells. Acta Pharmacol Sin, 2008, 29: 614-619

19 Pulaski B A, Clements V K, Pipeling M R, et al. Immunotherapy with vaccines combining MHC class II/CD80+ tumor cells with interleukin-12 reduces established metastatic disease and stimulates immune effectors and monokine induced by interferon gamma. Cancer Immunol, 2000, 49: 34-45

20 Michigami T, Hiraga T, Williams P J, et al. The effect of the bisphosphonate ibandronate on breast cancer metastasis to visceral organs. Breast Cancer Res Treat, 2002, 75: 249-258

21 Hiraga T, Ueda A, Tamura D, et al. Effects of oral UFT combined with or without zoledronic acid on bone metastasis in the 4T1/luc mouse breast cancer. Int J Cancer, 2003, 106: 973-979

22 Yang J, Mani S A, Donaher J L. Twist, a master regulator of morphogenesis, plays an essential role in tumor metastasis. Cell, 2004, 117: 927-939

23 Talia S F, Jeffrey D M, Mark E B, et al. The economic burden of metastatic breast cancer: A systematic review of literature from developed countries. Cancer Treatment Rev, 2011, 37: 405-415

24 Aslakson C J, Miller F R. Selective events in the metastatic process defined by analysis of the sequential dissemination of subpopulations of a mouse mammary tumor. Cancer Res, 1992, 52: 1399-1405

25 Lelekakis M, Moseley J M, Martin T J, et al. A novel orthotopic model of breast cancer metastasis to bone. Clin Exp Metastasis, 1999, 17: $163-170$

26 Park S J, Kim R J, Nam J S. Inhibitor of DNA-binding 4 contributes to the maintenance and expansion of cancer stem cells in $4 \mathrm{~T} 1$ mouse mammary cancer cell line. Lab Anim Res, 2011, 27: 333-338

27 Bao L, Haque A, Jackson K, et al. Increased expression of P-glycoprotein is associated with doxorubicin chemoresistance in the metastatic 4T1 breast cancer model. Am J Pathol, 2011, 178: 838-852

28 Jiang P D, Zhao Y L, Deng X Q, et al. Antitumor and antimetastatic activities of chloroquine diphosphate in a murine model of breast cancer. Biomed Pharmacother, 2010, 64: 609-614

29 Li J, Dong X, Xu Z, et al. Endostatin gene therapy enhances the efficacy of paclitaxel to suppress breast cancers and metastases in mice. $\mathbf{J}$ Biomed Sci, 2008, 15: 99-109

30 Zhen H Y, Xue Y, Zhen Y S. Inhibition of angiogenesis by antitumor antibiotic C-1027 and its effect on tumor metastasis. Natl Med J Chin, 1997, 77: 657-660

31 Huang Y H, Shang B Y, Zhen Y S. Antitumor efficacy of lidamycin on hepatoma and active moiety of its molecule. World J Gastroenterl, 2005, 11: 3980-3984

32 Chen J, Ouyang Z G, Zhang S H, et al. Down-regulation of the nuclear factor- $\kappa \mathrm{B}$ by lidamycin in association with inducing apoptosis in human pancreatic cancer cells and inhibiting xenograft growth. Oncol Rep, 2007, 17: 1445-1451

33 Zhen Y Z, Lin Y J, Shang B Y, et al. Enediyne lidamycin induces apoptosis in human multiple myeloma cells through activation of p38 mitogen-activated protein kinase and c-Jun NH2-terminal kinase. Hematology, 2009, 90: 44-51

34 Guo X F, Zhu X F, Shang Y, et al. A bispecific enediyne-energized fusion protein containing ligand-based and antibody-based oligopeptides against epidermal growth factor receptor and human epidermal growth factor receptor 2 shows potent antitumor activity. Clini Cancer Res, 2010, 16: 2085-2094

35 Li L, Huang Y H, Li Y, et al. Antitumor activity of anti-type IV collagenase monoclonal antibody and its lidamycin conjugate against colon carcinoma. World J Gastroenterol, 2005, 11: 4478-4483

36 Kennedy D R, Beerman T A. The radio mimetic enediyne C-1027 induces unusual DNA damage responses to double-strand breaks. Biochemistry 2006, 45: 3747-3754

37 He Q Y, Liang Y Y, Wang D S, et al. Characteristics of mitotic cell death induced by enediyne antibiotic lidamycin in human epithelial tumor cells. Int J Oncol, 2002, 20: 261-266

Open Access This article is distributed under the terms of the Creative Commons Attribution License which permits any use, distribution, and reproduction in any medium, provided the original author(s) and source are credited. 